\title{
Synchronisation of spatiotemporal complex states by incoherent coupling
}

Katharina Havermann

k.havermann@uni-muenster.de

Björn Gütlich

Cornelia Denz

denz@uni-muenster.de
Institut für Angewandte Physik and Center for Nonlinear Science, Westfälische Wilhelms-Universität Münster, Corrensstrasse 2-4, 48149 Münster

Institut für Angewandte Physik and Center for Nonlinear Science, Westfälische Wilhelms-Universität Münster, Corrensstrasse 2-4, 48149 Münster

Institut für Angewandte Physik and Center for Nonlinear Science, Westfälische Wilhelms-Universität Münster, Corrensstrasse 2-4, 48149 Münster

Synchronisation of spatiotemporal continuous disorder is realised in a Liquid Crystal Light Valve single feedback system with an incoherent, unidirectional master-slave-coupling scheme as excellent model system for synchronisation. Thus, complex states disordered in space and time were completely synchronised by using identical systems as master and slave. Thereby the impeding role of system differences is demonstrated in comparison to former experiments. A novel imaging method is introduced, in which the synchronisation process and effects like a time lag can be more easily characterised. [DOI: 10.2971/jeos.2008.08001]

Keywords: Synchronisation, nonlinear dynamics, liquid crystals, spatiotemporal pattern formation

\section{Introduction}

Synchronisation is a fascinating phenomenon with a great amount of impressive examples in biology, chemistry, sociology and various other fields [1]. In addition to the understanding of synchronisation, especially the creation and use of chaos synchronisation has become a topic of great interest [2], particularly the chaos based encryption via optical chaotic systems is a strong motivation for investigation [3]. Thus, it is well known that the chaotic behaviour of nonlinear oscillators can be synchronised in the time domain [4]. The research on the coupling of two chaotic oscillators, bidirectional schemes, and complex networks recently gave rise to the investigation of spatiotemporal continuous systems [5]-[7].

In contrast to synchronisation of one dimensional signals, continuous spatial extended systems are both theoretically and experimentally more challenging and particularly in experiments spatiotemporal chaos synchronisation is rather uninvestigated. Although some spatiotemporal complex systems can be locally coupled, e.g. reaction diffusion systems in chemistry, nonlinear optical systems with their easy tunable system parameters and precisely controllable coupling conditions offer great experimental possibilities. The LCLV single feedback system holds an outstanding position in experiment. Together with a wide range of accessible dynamics time constants of a few milliseconds allow a detailed analysis of the behaviour. That is why for the examination synchronisation of optical systems our scheme is appealing as simple and easy influenceable model. If it was possible to completely understand and use synchronisation of spatiotemporal complex states, encrypted data processing of two dimensional digital or analog data arrays can principally be realised very fast without an electronic interface.
Complete synchronisation is possible within the chaotic regime for identical systems only [1]. Therefore, one of the greatest challenges when investigating synchronisation properties of various systems is the issue of equality of system parameters. For systems continuous in space differences principally can evolve in every single point in space. Therefore, especially spatial non-uniformities need to be considered [8].

Pioneering experiments in a Liquid Crystal Light Valve [LCLV] single feedback system as model system demonstrated the principle of experimental spatiotemporal synchronisation in a master-slave-configuration, where two subsystems approximate two identical systems [9]. There we could show that synchronisation can be experimentally realized in this system. However, only a low synchronisation state of $C=0.4$ could be reached. However, as complete synchronisation is a crucial condition for chaos communication, in this contribution, we are able to exclude every system inherent difference between master and slave using an incoherent coupling, thereby allowing for the first time to our knowledge to reach complete synchronisation. All degrees of dynamics are investigated and the synchronisation process is studied multifariously with a novel imaging technique.

In the following chapters we introduce the LCLV single feedback system, then the coupling scheme and the novel calibration method are described. Subsequently the novel imaging technique for synchronisation is introduced. Finally, due to the exact calibration method, our results are presented via this new imaging method. 


\section{INCOHERENT SYNCHRONISATION OF SPATIOTEMPORAL DISORDER}

The unidirectional, incoherent coupling scheme includes an LCLV single feedback system and an external control unit. At first, the LCLV single feedback system ist introduced and its pattern forming properties and the transition to spatiotemporal disorder is described. Subsequently the coupling scheme is regarded in detail.

\subsection{LCLV single feedback system}

In order to realize a system that is able to demonstrate synchronisation of spatiotemporal disorder, a system exhibiting complex spatiotemporal behaviour is necessary. Nonlinear single feedback systems do not only represent a simple configuration that already exhibits fully developed spatiotemporal dynamics, but represent a class of systems in which the mechanism of pattern formation and the formation of spatiotemporal disordered behaviour are by now well understood [10, 11]. They combine nonlocal coupling and a nonlinear medium most elementary. The single feedback configuration considered here uses a LCLV as nonlinearity, which possesses the characteristics of a saturable Kerr type [12].

An LCLV consists of a liquid crystal layer and a photo conductive layer, which are separated by a dielectric mirror. When applying a DC voltage to the transparent, unstructured electrodes an intensity modulation incident on the photo conductive side, also denoted as write side of the LCLV, is effectively transformed into a phase shift of the light passing the read side. The write light with the local intensity $I_{w}$ changes the local impedance of the photo conductive layer, which affects the internal electric field and therefore the alignment of the liquid crystals. This again results in a refractive index change at the LC layer. Due to the refractive index modulation, the phase of the light, passing the LC layer, which is also called read out side, is affected. The phase transformation can be written as a nonlinear differential equation of the phase $\phi$

$$
\begin{aligned}
\tau \dot{\phi}-l^{2} \Delta_{\perp}^{2} \phi & +\phi \\
& =\phi_{\max }\left[1-\tanh ^{2}\left(\frac{1+\kappa_{\tau} I_{f} S_{0}}{1+\kappa_{S} I_{f} V_{0}} \cdot V_{\text {ext }}-\frac{V_{t h}}{V_{0}}\right)\right]
\end{aligned}
$$

with the phase $\phi$, the diffusion length $l$, the external applied voltage $V_{\text {ext }}$, the response time $\tau$, the feedback intensity at the write side $I_{f}$ and the propagation length $L . \kappa_{\tau}, \kappa_{s}, S_{0}$ and $v_{0}$ are device specific fit parameters. Because of the reflective configuration of the LCLV, a ring-like geometry is used for single feedback, as shown in Figure 1. The nonlocal feedback mechanism needed to induce selforganised pattern formation is caused by a distance $\mathrm{L}$ of free space propagation. Due to the propagation the phase distribution is transformed into an amplitude distribution as a result of diffraction. By formal integrating the field equation describing the free propagation, the field of the propagated light can be written as

$$
\mathbf{E}_{\mathbf{f}}=\exp \left[-\frac{L}{k_{\lambda}} \nabla_{\perp}^{2}\right] e^{i \phi} \mathbf{E}_{\mathbf{i}}
$$

The input beam is phase modulated by the liquid crystal layer of the LCLV, reflected at the internal mirror and again passes

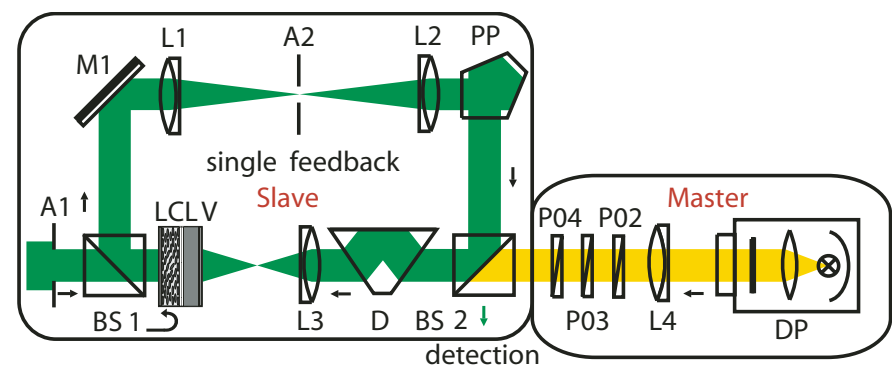

FIG. 1 Sketch of the experimental configuration to realise spatiotemporal synchronisation in a single feedback system. Left: single-feedback system acting as a slave system, including LCLV and feedback slope. Right: master system built by a data projector.

the liquid crystal layer, where it becomes modulated a second time. A 4-f-arrangement and an additional lens image the amplitude distribution onto the photo conductive layer of the LCLV. The system response is recorded by coupling out some intensity at beam splitter BS2.

The described system exhibits a modulation instability, i.e. the growth of spatial patterns out of the uniform solution above a certain threshold intensity $[13,14]$. In this system, the formation of hexagonal, i.e. periodic patterns is observed directly above threshold. The period of these periodic structures is depending on the wave vector of the laser light $k_{0}$ and the propagation length $L$ expressed by the transverse critical wave vector $k_{c}=\sqrt{2 \pi k_{0} / 3 L}$, available from a linear stability analysis. With increasing pump intensity, higher order transverse wave vectors become excited, resulting in the breakdown of the spatial order. Due to the competition of modes in addition to the loss of spatial order temporal dynamics sets in, which is often denoted as spatiotemporal complexity or space time chaos [15]. In Figure 2 the development of the space time dynamics is illustrated. From the left to the right the write intensity in-

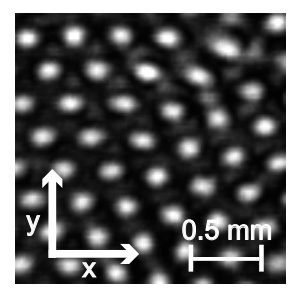

(a)

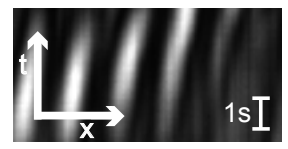

(d)

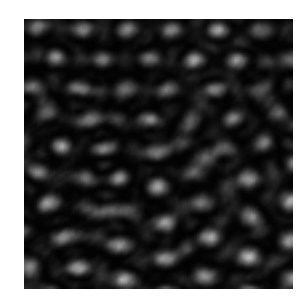

(b)

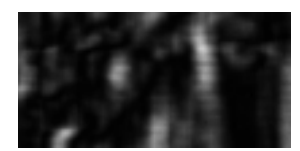

(e)

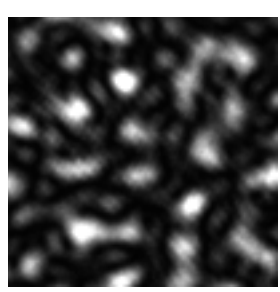

(c)

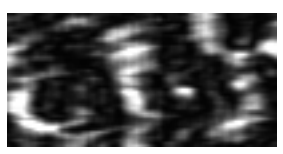

(f)
FIG. 2 System behaviour in space (a-c) and time (d-f), from left to right: increasing intensity $I_{w}: 3 I_{t h r}(\mathrm{a}, \mathrm{d}), 5 I_{t h r}$ (b, e), $12_{t h r}$ (c, f) top: development of spatial disorder with increasing write intensity; video shows spatiotemporal behaviour (size 200kB, format: avi, see Figza.avi, Fig2b.avi, Fig2c.avi) ; bottom: $x$-axis: cross section along $x$-axis, $y$-axis: time.

creases from the minimum intensity, at which spatiotemporal disorder evolves, to maximum reachable dynamics. The left Figures $2 \mathrm{a}$ and $2 \mathrm{~d}$ are at a regularly patterned state at $3 I_{t h r}$, followed by a medium dynamic state with increased disorder at $5 I_{t h r}$ in Figures $2 \mathrm{~b}$ and $2 \mathrm{e}$. The Figures $2 \mathrm{c}$ and $2 \mathrm{f}$ on the right show illustrations of the space time chaos at $12 I_{t h r}$. The 
upper row of figures shows the image plane on the photo conductive layer. The initially regularly patterned states become more and more disordered finally showing highly spatiotemporal complex states. In the second row the temporal evolution is shown. One cross section through the image plane is tracked for a temporal sequence of 4.5 seconds duration and plotted along the y-axis. In the low dynamic case the pattern state remains constant during the whole measurement. In the medium dynamic case first temporal fluctuations of the emerging structures can be observed, while for the high intensity case the spatiotemporal disorder is fully evolved. Thus, the control parameter pump intensity, which adjusts the spatial dynamics of the system, also controls the temporal dynamics.

A supplementary control method is the use of spatial filtering in the Fourier plane of the $4 \mathrm{f}$-arrangement of the feedback loop. Therefore, the onset of spatiotemporal dynamics can be controlled with a low pass to cut off the transverse wave numbers above the first band. The evolving spatial and temporal disorder increases more smoothly with rising pump intensity when low pass filtered [16].

\subsection{Coupling scheme}

In the synchronisation scheme under consideration the coupling of identical systems is realised by recording the master and injecting it back into the system again. Thus, one LCLV single feedback system is able to act as master or slave system depending on the operational mode of the scheme. In the first mode the free running system is recorded in its role as master. In a second step the recorded master is coupled incoherently into the feedback system with the help of a data projector. The now coupled system is recorded as slave. Due to the time delay between the recording of master and slave only random correlations exist between uncoupled master and slave thus demonstrating the independence of the systems. As soon as an appropriate charactarisation method is derived in the next section this issue is discussed more thoroughly.

In previous experiments with two subsystems located on one LCLV a coupling via intensity was performed. The coupling was implemented by adding intensity from the master system into the slave system, while the master system is complemented with some bias intensity to keep the systems as equal as possible: $I_{\text {master }}^{\prime}=I_{\text {master }}+I_{\text {bias }}$ and $I_{\text {slave }}^{\prime}=I_{\text {slave }}+\gamma I_{\text {master }}$. The coupling strength was easily definable as $\gamma=\frac{I_{\text {master }}}{I_{\text {slave }}}$. At $\gamma=1$ master and slave intensities are equal.

In contrast, the use of incoherent projector light requires a definition of the coupling strength $\gamma$ by the average phase shift of the induced projector light. The use of lights from two generally different sources with different spectra, namely the white light of the data projector and the coherent laser light, makes it difficult to define the coupling via intensity because of the wavelength dependence of the photo conducting layer of the LCLV where the coupling in fact takes place. Therefore, a calibration of the dependence of the phase shift on the light intensity is required for both sources. The phase shift of the LCLV was measured in a standard double slit experiment by varying independently the write intensity $I_{w}$ on the photo conductive layer and the read out intensity of the pump beam $I_{p}$ [17]. One half of the photo conductive layer is irradiated by the projector light or by the laser light respectively thus inducing a refractive index change at one side of the LC layer. When the induced profile is read out and the double slit is irradiated with the resulting field, the light field incident at one of the slits experiences an extra phase shift due to the irradiation of the photo conductive layer. The phase shift difference between both parts of the LCLV is detected by the offset of the stripe pattern behind the double slit. During the calibration process an additional dependence on the pump intensity $I_{p}$ at the liquid crystal side was found. In Figure 3 the measured phase shift for the laser light (Figure 3a) and the incoherent projector light (Figure $3 \mathrm{~b}$ ) is shown. Along the x-axis the intensity $I_{p}$
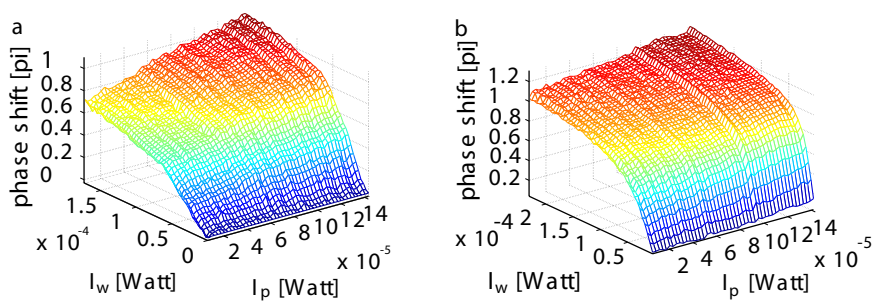

FIG. 3 Phase shift in units of $\pi$, x-axis: intensity on LC layer, $y$-axis: intensity on photo conductive layer, (a) laser light induced phases shift, (b) projector light induced phase shift.

on the LC layer is varied, while along the y-axis the intensity on the photo conducting layer $I_{w}$ is changed. A strong dependence on the intensity $I_{w}$ at the photo conductive layer is measured which directly demonstrates the difference between the optical way of the unaffected and the reoriented liquid crystals. The general behaviour of the phase shift is a saturating monotonously increasing function, whereas the phase shift induced by the projector light after a sharper rise saturates earlier. Furthermore a slowly increasing dependence is detected along the $\mathrm{x}$-axis for both light sources, i.e. for rising pump intensity $I_{p}$ the phase shift is increasing. The dependence of the phase shift on the pump intensity at the read out side is caused by an imperfect reflectivity of the internal mirror of the LCLV. The transmitted light of the pump beam causes an additional effect on the photo conductive layer and a shift in the refractive index change of the LC layer occurs. This effect is revealed to be constant when comparing the phase shifts of the different light sources and a calibration curve for the whole range of system intensities was found. In Figure 4 the phase shift difference scaled to the phase shift of the laser light is depicted. For better illustration the y-axis, namely the varied intensity at the read out layer is reversed. The stronger noise for smaller pump intensity at the LC layer and higher write intensity at the photo conductive layer is due to the fact that the intensity at the LC layer simultaneously forms the detected signal. Thus, for vanishing intensity at the LC layer the measurement signal vanishes.

After identifying the corresponding correction factor for the intensity dependent coupling the coupling strength $\gamma=$ $\frac{2 \phi_{\text {master }}}{\phi_{\text {free }}}$ can be realised. The factor of two is added to keep $\gamma=1$ for equal fractions of master and slave. As described before the system behaviour strongly depends on the pump intensity. To accomplish the preservation of a constant operation 


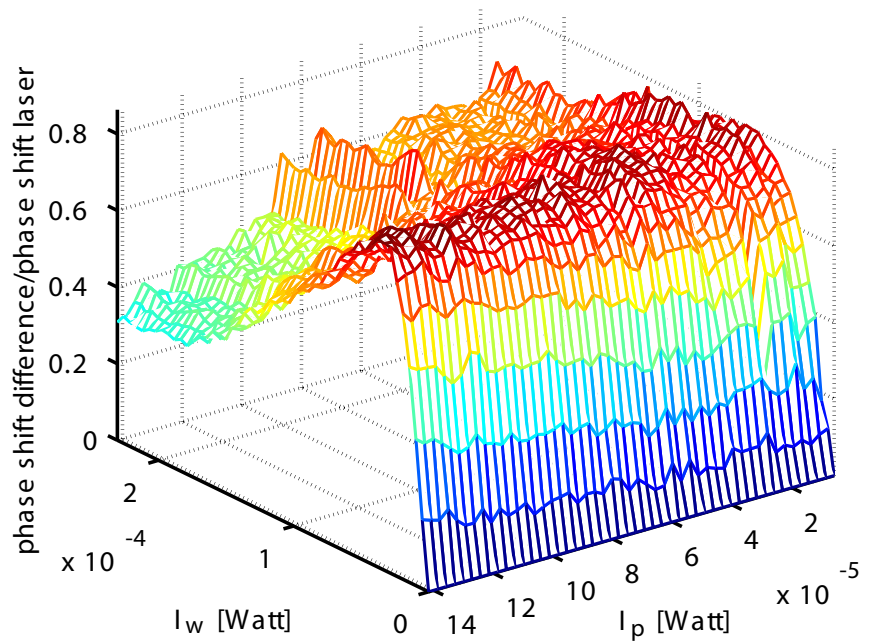

FIG. 4 Resulting measured phase shift difference between both sources, normalised to laser intensity.

point and to reach optimal conditions for synchronisation it is essential to keep the average phase shift in the system fixed. By reducing the intensity of the master in the same manner it is added by the coupling signal the operation point is preserved. Together with the knowledge of the intensity dependence on the phase shift measured before, a standard ansatz

$$
\left\langle\phi_{\text {slave }}\right\rangle=(1-\gamma)\left\langle\phi_{\text {free }}\right\rangle+\gamma\left\langle\phi_{\text {master }}\right\rangle,
$$

is possible, were no equivalent to the bias intensity is needed.

\section{SPATIOTEMPORAL SYNCHRONISATION}

In the following chapter the synchronisation measurements are described with the help of correlation planes. These diagrams include information of the synchronisation process as well as of the decoherence time and random correlations. Using the calibration curve measurements of a novel exactness are performed in the whole range of dynamics -from slightly above the periodic state up to the highly dynamic range.

\subsection{Realisation of unidirectional coupling scheme}

In the measuring process the free running system is recorded (Figure 2a). After a sufficient time to exclude correlations between master and slave this information is fed back into the system by the data projector. The transmitted white light distribution of the data projector is injected into the system by a beam splitter, and directly imaged on the photo conductive layer of the LCLV, thus realising the coupling strength $\gamma$ by an arrangement of three polarisers. Over the whole range of coupling strength for one set of measurements one single master is used. Three measurements are performed over about three seconds for each coupling strength.

Master and slave dynamics are compared at certain points of time to analyse the synchronising behaviour of the spatiotemporal disorder. An adequate measure for synchronisation is the cross correlation [9]

$$
\left\langle C_{A B}(\Delta \mathbf{x}, \Delta t, t)\right\rangle=\frac{\left\langle\tilde{I}_{A}(\mathbf{x}, t) \cdot \tilde{I}_{B}(\mathbf{x}-\Delta \mathbf{x}, t-\Delta t)\right\rangle}{\sqrt{\left\langle\tilde{I}_{A}^{2}(\mathbf{x}, t)\right\rangle_{x}\left\langle\tilde{I}_{B}^{2}(\mathbf{x}, t)\right\rangle_{x}}}
$$

To avoid effects of the margins, a central part of the whole aperture is chosen. The depiction of a plane of correlation of all existing combinations of master and slave dynamics turned out to give a visual understanding of the synchronisation process. Higher correlations should emerge for the diagonal of simultaneous existence of master and slave due to synchronisation, while for nonzero time differences between master and slave only random correlations should exist. In Figures $5 \mathrm{~d}, 5 \mathrm{e}$, and $5 \mathrm{f}$ master and slave are correlated at certain points of time for zero coupling for all possible time differences in the measuring process. Random correlations up to
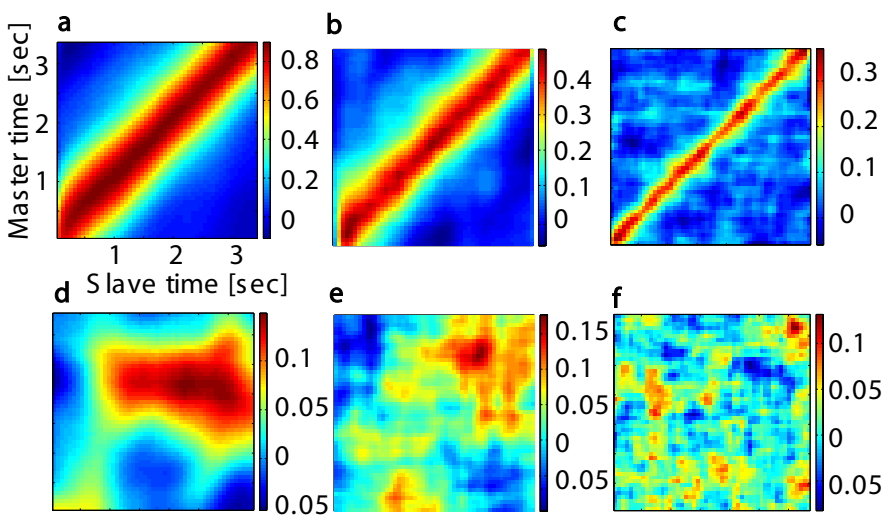

FIG. 5 The correlation is plotted versus master and slave in a false colour representation, (a) slow dynamics, correlated; (b) medium dynamics, correlated; (c) high dynamics, correlated, (e-f) corresponding cases for uncorrelated systems.

about 0.15 appear due to the restricted set of possible transverse wave vectors. In addition to the comparison of the spatial distribution, also the temporal dynamics can be quantified out of this plots. The texture of the random structures in the correlation-planes is a measure for the dynamics in the system and can be interpreted as the decoherence time of the random correlation. In Figures 5a, 5b, and 5c the correlation plane for the same degrees of dynamics is shown for non vanishing coupling. A diagonal is clearly visible, which indicates the onset of spatiotemporal synchronisation. Phenomena like time lags can easily be detected and also excluded. In case of a constant time lag a shift of the diagonal line of maximum correlation was observable. Furthermore, the width of the diagonal correlation line marks the decorrelation time.

\subsection{Characterisation of spatiotemporal synchronisation}

An overview of the experimental results is shown in Figure 6, where the correlation depending on the coupling strength is displayed for measurements from the slightly dynamic regime up to strong dynamics at $10 I_{t h r}$. The measurements at $3 I_{t h r}, 5 I_{t h r}$ and $10 I_{t h r}$ were performed with low pass filtering higher modes. To demonstrate, that this is not a necessary criteria, a reference measurement without low pass filtering was performed. One data point consists of the average of three measurements. The general dependence of the degree of synchronisation on the coupling strength is equal for all degrees 


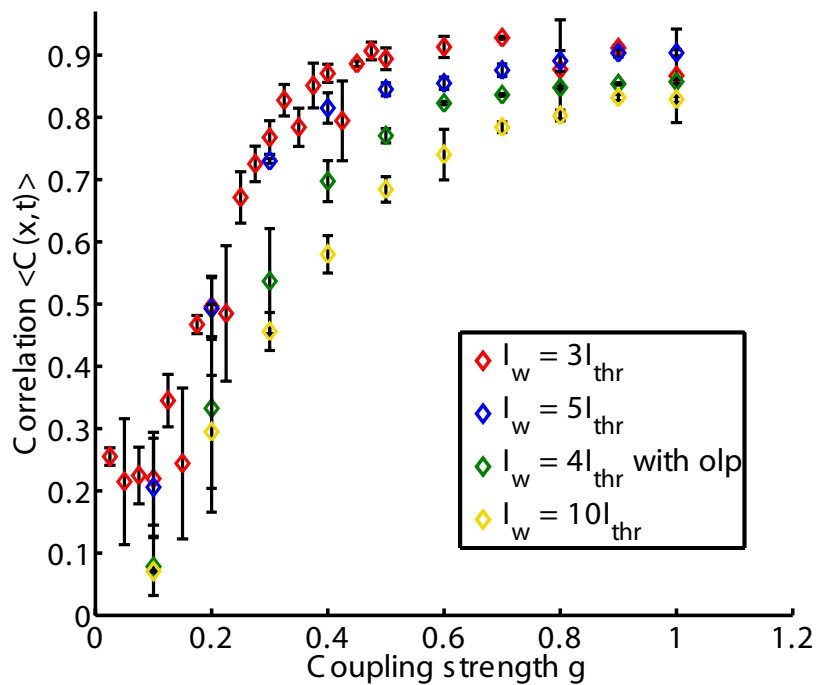

FIG. 6 Synchronisation measurements depending on the coupling strength for the whole range of dynamic, at $I_{w}=4 I_{t h r}$ with open low pass.

of dynamics and shows a monotonously increasing function which saturates for high coupling constants. The inclination of the curves slightly depends on the dynamics in the system. The coupling strength $\gamma$, sufficient to reach maximum synchronisation, is monotonously dependent on the degree of dynamics, which was increased by rising system intensity and for $4 I_{t h r}$ by omitting the low pass filtering.

The development of a synchronisation process can be observed in detail in its correlation plane. To discover the mechanisms suppressing synchronisation for smaller coupling strengths two correlation planes are regarded, at first a weakly dynamic case, the second one with strong dynamics. In Figure 7a an example with low dynamics at a write intensity of $I_{w}=3 I_{t h r}$ is shown at a coupling strength of $\gamma=0.5$, next to the correlation curve of the same measurement with time on the $x$-axis and the correlation on the y-axis in Figure $7 \mathrm{~b}$. As we can see in Figure 6 this measurement is
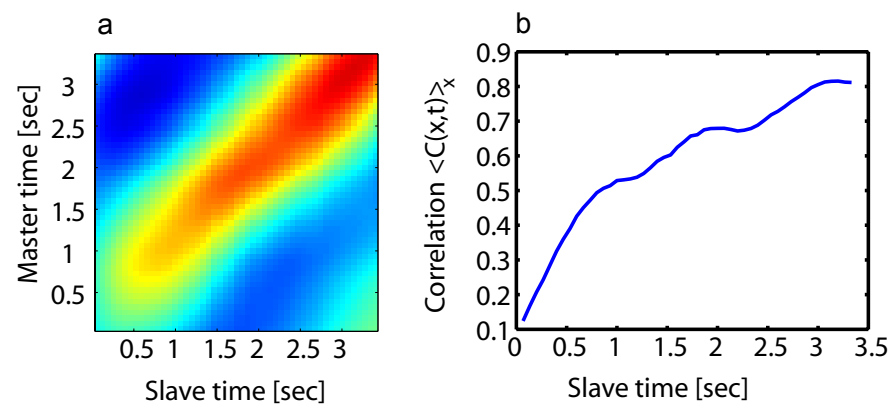

FIG. 7 Slow rise of synchronisation at write intensity $3 I_{t h r}$, coupling strength $\gamma=0.5$, the correlation continues to increase after 3 seconds, (a) the correlation is plotted versus master and slave in a false colour representation, $x$-axis: slave time, $y$-axis: master time; (b) plot of the maximum correlation along the diagonal of (a), $x$-axis: slave time, y-axis: maximum correlation.

close to the saturation of the synchronisation state. A slow rise of synchronisation is detected, which is not finished yet at the end of the measurements after three seconds. The temporal evolution of synchronisation is thus dependent on the coupling strength. For vanishing coupling strength the time needed to reach maximum synchronisation becomes infinite. When can conclude, that even for high coupling strengths the synchronisation time amounts to a multiple of the decorrelation time. In a highly spatiotemporal complex system, this slow process can easily be interrupted by fluctuations due to noise. Therefore, the increase of synchronisation is damped and even collapses can be detected. In Figure 8a a synchronisation process at an intensity of $I_{w}=10 I_{t h r}$ with a coupling of $\gamma=0.5$ is shown. The synchronisation rises slowly and is interrupted several times due to the dynamics including a temporal break down of synchronisation after about five seconds, as one can also recognise in the adjacent synchronisation graph in Figure $8 \mathrm{~b}$. Therefore, interruptions of the synchronisation process can be hold responsible for their slower rise at strong dynamics.
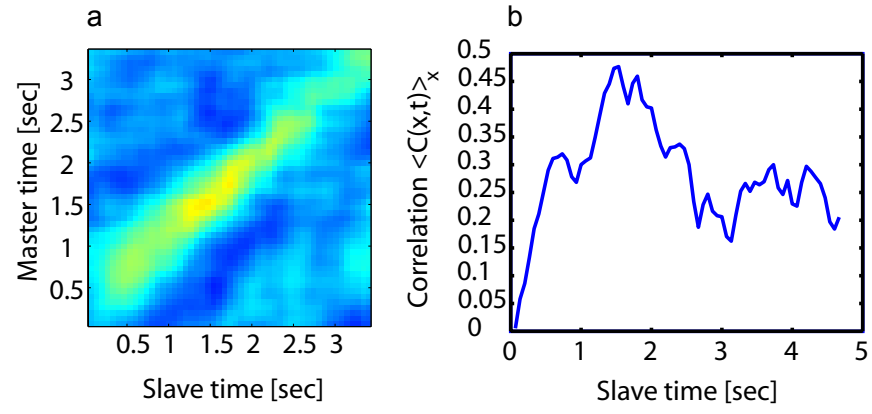

FIG. 8 Collapse of synchronisation due to complex spatiotemporal system dynamics, at a write intensity $I_{w}=10 I_{t h r}$, coupling strength $\gamma=0.5$; (a) the correlation is plotted versus master and slave in a false colour representation, $x$-axis: slave time, $y$-axis: master time, (b) plot of the maximum correlation along the diagonal of (a), $x$-axis: slave time, $y$-axis: maximum correlation.

In Figure 9 the rise of synchronisation when increasing the coupling constant is illustrated in movies of the correlation planes. One video consists of correlation planes for a definite

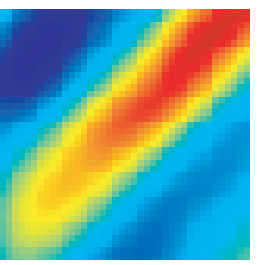

(a)

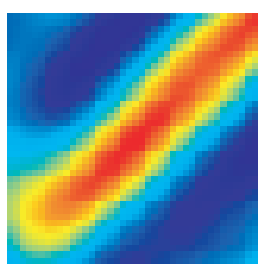

(b)

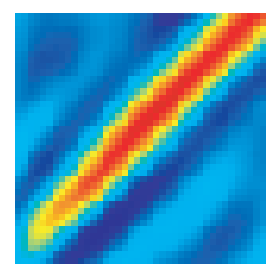

(c)

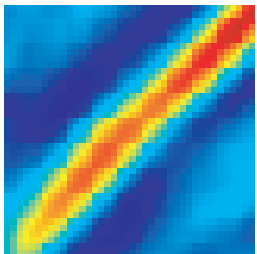

(d)

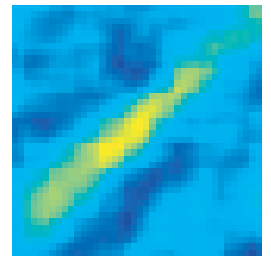

(e)
FIG. 9 Correlation planes in a false colour representation, for all degrees of dynamics, coupling strength $\gamma=0.5$, x-axis: Slave time, $y$-axis: Master time, write intensities from left to right: $3 I_{t h r}, 4 I_{t h r}, 5 I_{t h r}, 7 I_{t h r}, 12 I_{t h r}$; movie: coupling strength $\gamma$ from 0.1 to 1.0 (videosize: 30kB, format: avi, see Figga.avi, Figgb.avi, Figgc.avi, Figgd.avi, Figge.avi).

state of dynamics, while the coupling strength increases dur- 
ing the progress of the movie. From left to right the write intensity is rising and the synchronisation processes for all degrees of dynamics are opposed. The exposed dependence of the increasing of synchronisation on the dynamics of the spatiotemporal complex states is observable as well as the decoherence time, perceivable from the thickness of the synchronisation diagonal. A time lag was not detected in this measurements. Apart from the detailed analysis of the synchronisation process, the measurement contain another novelty. It is clearly visible, that complete synchronisation can be reached for all degrees of dynamic. Even for the highly dynamic range, which is in the strongly spatiotemporal disordered regime, for higher coupling constants complete synchronisation was detected, becoming manifest in correlations of $\langle C\rangle=0.9$.

\section{CONCLUSION}

In conclusion, we have shown that high degrees synchronisation of strongly dynamic spatiotemporal complex states is reachable in an unidirectionally coupled master-slave configuration. By realising an LCLV-single feedback system with an external control unit as a spatiotemporally extended masterslave system, we could reach high correlations up to $\langle C\rangle=$ 0.9 , which allow the denomination as complete synchronisation. In contrast to earlier experiments with two subsystems, synchronisation of two unidirectionally, incoherently coupled identical systems reaches unity for all degrees of complexity. Therefore, the strong influence of system differences was demonstrated. This results give reason to consider the future use of spatiotemporal systems for the suggested chaos encrypted communication scheme.

\section{References}

[1] A. Pikovsky, M. Rosenblum, and J. Kurths, Synchronization (Cambridge University Press, 2001).

[2] S. Boccaletti, J. Kurths, G. Osipov, D. L. Valladares, and C. S. Zhou, "The synchronization of chaotic systems" Phys. Rep. 366, 1(2002).

[3] J. Garcia-0jalvo and R. Roy, "Spatiotemporal Communication with Synchronized Optical Chaos" Phys. Rev. Lett. 86, 5204 (2000).
[4] I. Fischer, Y. Liu, and P. Davis, "Synchronization of chaotic semiconductor laser dynamics on subnanosecond time scales and its potential for chaos communication" Phys. Rev. A 62, 011801 (2000).

[5] R. Neubecker, B. Thüring, M. Kreuzer, and T. Tschudi, "Pattern formation in nonlinear optical systems" Chaos Soliton. Fract. 10, 681 (1999).

[6] G. Hu, J. Xiao, J. Yang, F. Xie, and Z. Qu, "Synchronization of spatiotemporal chaos and its applications" Phys. Rev. E 56, 2738 (1997).

[7] A. A. Koronovskii, P. V. Popov, and A. E. Hramov, "Ceneralized Chaotic Synchronization in Coupled Ginzburg-Landau Equations" 103, 654 (2006).

[8] R. Neubecker, E. Benkler, R. Martin, and G. L. Oppo, “Manipulation and Removal of Defects in Spontaneous Optical Patterns" Phys. Rev. Lett. 91, 113903 (2003).

[9] R. Neubecker and A. Zimmermann, "Spatial forcing of spontanous optical patterns" Phys. Rev. E 65, 035205(R) (2002).

[10] W. J. Firth, "Spatial instabilities in a Kerr medium with single feedback mirror" J. Mod. Optic. 37, 151 (1990).

[11] R. Neubecker, G.-L. Oppo, B. Thüring, and T. Tschudi, "Pattern formation in a liquid-crystal light valve with feedback, including polarisation, saturation and internal threshold effects" Phys. Rev. A 52, 791-808 (1995).

[12] R. Neubecker, G. L. Oppo, B. Thuering, and T. Tschudi, "Pattern formation in a liquid-crystal light valve with feedback, including polarization, saturation, and internal threshold effects" Phys. Rev. A 52, 791 (1995).

[13] P. L. Ramazza, S. Ducci, S. Boccaletti, and F. T. Arecchi, "Localized versus delocalized patterns in a nonlinear optical interferometer" J. Opt. B 2, 399 (2000).

[14] M. C. Cross and P. C. Hohenberg, "Pattern formation outside of equilibrium" Rev. Mod. Phys. 65, 851 (1993).

[15] G. Schliecker and R. Neubecker, "Voronoi analysis of the breakdown of order in spontaneous optical spot patterns" Phys. Rev. E. 61, R997 (2000).

[16] M. L. Berre, A. S. Patrascu, E. Ressayre, and A. Tallet, "Localized structures in chaotic patterns: From disorder to ordering" Phys. Rev. A 56, 3150 (1997).

[17] M. Born and E. Wolf, principles of optics (Pergamon Press, 1964). 